\title{
Moderate-dose atorvastatin improves arterial endothelial function in patients with angina pectoris and normal coronary angiogram: a pilot study
}

Amela Kabaklić ${ }^{1}$, Zlatko Fras ${ }^{2,3}$

${ }^{1}$ Department of Hypertension, University Medical Centre Ljubljana, Ljubljana, Slovenia ${ }^{2}$ Centre for Preventive Cardiology, Department of Vascular Medicine, Division of Medicine, University Medical Centre Ljubljana, Ljubljana, Slovenia

${ }^{3}$ Chair of Internal Medicine, Medical Faculty, University of Ljubljana, Ljubljana, Slovenia

Submitted: 17 September 2016

Accepted: 8 November 2016

Arch Med Sci 2017; 13, 4: 827-836

DOI: https://doi.org/10.5114/aoms.2017.68238

Copyright $\odot 2017$ Termedia \& Banach

\section{Abstract}

Introduction: Endothelial dysfunction could contribute to the pathophysiology of angina pectoris (AP) in patients with normal coronary angiograms. Besides lipid-lowering effects, statins exert pleiotropic effects including improving endothelial function.

Material and methods: Our double-blind study included 58 patients with AP, noninvasively confirmed myocardial ischemia and a normal coronary angiogram. The effect of once-daily $20 \mathrm{mg}$ atorvastatin (A) was compared with placebo (P) for 6 months. Endothelial function was evaluated by flow-mediated dilation (FMD) of the brachial artery, and microcirculation by peripheral arterial tonometry (EndoPAT) measuring the reactive hyperemia index (RHI), indicating microcirculatory endothelial function, and the augmentation index (AI), an indicator of arterial stiffness. The impact of AP on the quality of life was monitored using the Seattle Angina Questionnaire (SAQ).

Results: Brachial artery endothelial dysfunction was found in $91.4 \%$ of patients at study entry, and subnormal RHI in $41 \%$. Group A showed an improvement of FMD compared with group P, both at 3 and 6 months $(+120.8 \%$ vs. $-21.2 \%$, and $+70.8 \%$ vs. $-1.9 \%$, respectively, $p<0.001)$. No difference was detected in the RHI. Rate-normalized Al showed an improvement $(-114.49 \%$ group A vs. $-30.77 \%$ group $P, p=0.077$ ), although the differences between the groups were not significant. According to the SAQ, an improvement was found in almost all observed variables with the exception of the issue of quality of life $(\mathrm{Q} o \mathrm{~L})$, where patients in both groups assessed their QoL at the control study visits as poorer compared with baseline.

Conclusions: Moderate-dose atorvastatin therapy improves endothelial function of large conduit arteries in patients with AP and a normal coronary angiogram, which probably reflects positive effects on coronary artery endothelial function. No effect was found with vascular effects at the level of the peripheral microcirculation.

Key words: microvascular angina, endothelial function, atorvastatin.

\section{Introduction}

Angina pectoris (AP) is a clinical manifestation of myocardial ischemia due to reduced coronary perfusion reserve. Most often it is the result of stenotic atherosclerotic changes in the epicardial

\author{
Corresponding author: \\ Prof. Zlatko Fras MD, PhD, \\ FESC, FACC \\ Division of Medicine \\ University Medical \\ Centre Ljubljana \\ Zaloška 7 \\ SI-1525 Ljubljana, Slovenia \\ Phone: +3861522 3152 \\ Fax: +38615223118 \\ E-mail: zlatko.fras@kclj.si
}


coronary arteries, while a normal angiogram and/or non-obstructive stenosis is detected in 20-30\% of patients with AP and previously noninvasively detected myocardial ischemia [1-3]. The causes of chest pain in these patients in clude either epicardial artery disease (endothelial dysfunction, coronary artery spasm, muscle bridge), microvascular coronary dysfunction and/or noncoronary disease [4]. Ischemia probably occurs because of irregularly distributed and inappropriate constriction of periarteriolar blood vessels across the myocardium. A functional disorder of the coronary microcirculation can ensue as insufficient vasodilatation and/or hypersensitive vasoconstriction to various metabolic and pharmacological stimuli [1, 2].

Endothelial dysfunction is one of the earliest indicators of deterioration of the vessel wall and is a good indicator of atherosclerosis development $[5,6]$. It creates a suitable condition for the activation and adhesion of leukocytes and platelets as well as the activation of cytokines, which increas es the permeability of the vessel wall for oxidized lipoproteins and inflammatory mediators. This results in a structural change of the arterial wall with the proliferation of smooth muscle cells and the formation of plaques [7, 8].

Hypercholesterolemia as the most important risk factor for coronary atherosclerosis is associated with deterioration of the endothelium-dependent vasodilation [9]. It is widely recognized that statins, currently the most commonly used lipid-lowering drugs, exert several so-called pleiotropic effects on the arterial wall. These include nitric oxide synthase (NOS) activation to improve endothelial function, lowering plasma endothelin-1 (a vasoconstrictor) and asymmetrical dimethylarginine (endogenous NOS inhibitor) levels, and inhibiting inflammation as well as different antithrombotic and antioxidant effects contributing to atherosclerotic plaque stabilization [10-14]. Beneficial effects of atorvastatin on endothelial function have been demonstrated not only in patients with normal myocardial function but also in patients with heart failure and dilated cardiomyopathy [15-18].

The aim of our study was to determine whether treatment with a moderate dose of atorvastatin improves endothelial function and reduces the burden of AP in patients with AP and angiographically normal or non-significantly stenosed coronary arteries.

\section{Material and methods}

\section{Patients and methods}

Our study included 58 patients $(31 \%, n=18$ males) with clinical manifestation of AP, in whom myocardial ischemia was confirmed by either positive treadmill exercise testing and/or evidence of reversible perfusion defects during exercise myocardial scintigraphy using single-photon emission computed tomography (SPECT) with Tc-99m tetrofosmin. Obstructive atherosclerotic disease (CAD) (> 50\% stenosis) was excluded by coronary angiography. All angiograms were analyzed by two highly experienced and independent senior interventional cardiology consultants.

The study was designed as a pilot, double-blind, placebo-controlled, randomized intervention study. After the initial assessment of endothelial function and predefined laboratory parameters, patients were randomly assigned to one of the two groups, treated either with $20 \mathrm{mg}$ of atorvastatin once daily (group A) or placebo (group P). The study drugs were produced and prepared by Lek Sandoz pharmaceutical company (from Ljubljana, Slovenia) and stored in the hospital pharmacy until the time of use.

In addition to the study drugs, all participants were intended to be treated with: (1) bisoprolol (dose: $0,1.25,2.5$ or $5 \mathrm{mg}$, adjusted to initial heart rate and blood pressure), (2) amlodipine (2.5 or $5 \mathrm{mg}$, adjusted to blood pressure), and, (3) acetylsalicylic acid $100 \mathrm{mg}$ once daily. There were altogether 4 patients ( 2 in group A and 2 in group P) who were not treated with bisoprolol since their initial heart rate was less than $55 \mathrm{~min}^{-1}$. In addition, they were free to use short-acting nitrates in case of acute AP; however, none of them used nitrates $24 \mathrm{~h}$ before the study visits. For the entire study period patients were not allowed to use any other medicines, vitamin and/or food supplements known to affect endothelial function.

Exclusion criteria were as follows: (a) lipidlowering drug treatment within one month before inclusion in the study, (b) diabetes mellitus, (c) presence of absolute contraindications to any of the study medications, (d) smoking, (e) clinical and/or laboratory signs of inflammation, (f) significant heart failure (functional class III-IV according to NYHA), (g) clinically important valve disease, including mitral valve prolapse, (h) previous myocardial infarction, (i) uncontrolled arterial hypertension, (j) clinically manifested hypothyroidism, (k) known muscular or neuromuscular disease, (l) gastrointestinal disease which may cause reduced absorption of drugs, (m) the use of the following medicines: corticosteroids, erythromycin, androgens, immunosuppressive drugs, estrogens, $(\mathrm{n})$ total cholesterol $>8.0$ $\mathrm{mmol} / \mathrm{l}$ or $<3.5 \mathrm{mmol} / \mathrm{l}$ and/or triglycerides $>4.5$ $\mathrm{mmol} / \mathrm{l}$, (o) serum creatinine $>120 \mu \mathrm{mol} / \mathrm{l}$, (p) serum creatine kinase activity $(C K)>3$ times upper normal limit, (q) levels of aspartate aminotrans- 
ferase (AST) and alanine aminotransferase (ALT) $>3$ times above the upper normal limit, $(r)$ pregnancy, (s) patients < 18 years of age, and (t) known extracardiac causes of chest pain.

All patients were familiarized with the purpose and course of the study and signed an informed consent form prior to inclusion.

Patients were followed for a total period of 6 months during which, after the inclusion study visit (SVO), two further study visits, with the same examinations as in the initial investigations, were scheduled, after 3 (SV1) and 6 months (SV2). The study was conducted according to the principles of the Declaration of Helsinki, and the Slovenian National Medical Ethics Committee approved the protocol.

Upon entry into the study the inclusion/exclusion criteria were assessed, the available past medical records reviewed, physical examination and anthropometric measurements performed, and the 12-lead ECG recorded. Detailed medical history with special emphasis on AP frequency and presence of other cardiovascular risk factors was obtained.

\section{Laboratory methods}

Blood samples for some basic hematological and biochemical laboratory parameters were obtained at each study visit between 7 and 11 am after at least $12 \mathrm{~h}$ of fasting; patients were also not allowed to drink coffee or to consume substances that could affect endothelial function for at least $24 \mathrm{~h}$ before the study visit.

\section{Investigation of endothelial function of large conduit arteries using ultrasound}

Both endothelium-dependent and -independent dilation were measured on the right brachial artery at each of the study visits using a high-resolution ultrasound machine (Aloka, Hitachi, Japan) with a $10 \mathrm{MHz}$ linear ultrasound probe using the technique of Celermajer et al. [19]. The baseline (resting) arterial flow was calculated by multiplying the velocity time integral of the Doppler flow signal by the heart rate and the vessel cross-sectional area. Endothelium-dependent (flow-mediated) dilation (FMD) was calculated as the change of the final diameter of the brachial artery during reactive hyperemia. Endothelium-independent nitroglycerin-mediated dilation (NMD) was calculated using the same principle after sublingual application of $0.4 \mathrm{mg}$ of glycerol trinitrate (GTN). Both methods are described in detail elsewhere [7]. The same examiner blinded regarding the study drugs and patients' laboratory results carried out all measurements.
Investigation of the endothelial-dependent functional capability of microcirculation using PAT

Peripheral arterial tonometry (PAT) enables plethysmographic recording of finger pulse wave amplitude (PWA), a measure of pulsatile volume changes. The reactive hyperemia index (RHI), which can be used as a marker of endothelial function at the level of the microcirculation, was calculated as the ratio of average PWA between post- and pre-occlusion [7]. The augmentation index (AI) reflecting arterial stiffness was calculated via pulse wave analyses of the PAT signal. In our study the EndoPAT 2000 (Itamar Medical Inc., Caesarea, Israel) device was used. This method is described in detail elsewhere [7].

\section{The Seattle Angina Questionnaire (SAQ)}

At all study visits the patients had to complete a Seattle Angina Questionnaire (SAQ). The SAQ quantifies 5 domains measuring the impact of angina on the patient's health status: physical limitation, angina stability, angina frequency, treatment satisfaction and quality of life. Scores are generated for each domain and are on a scale of 0-100, with 0 denoting the worst and 100 the best possible status.

\section{Statistical analysis}

Categorical variables are presented as numbers and percentages and continuous variables as average values. We applied the $\chi^{2}$ test or 2 -sided Fisher's exact test and Mann-Whitney $U$ test or $t$-test for categorical and continuous variables, respectively. A multivariate analysis was adjusted for all independent outcome predictors. To avoid variable selection caused by spurious correlations, only variables showing a relationship with the outcome (defined as $p<0.10$ in the univariate model) were included as potential predictors. We identified the final multivariate model for each major outcome using a backward stepwise approach. A 2-tailed $p$-value $<0.05$ was considered significant. Analyses were carried out using SPSS for Windows, version 22

\section{Results}

Clinical and demographic characteristics of the study population are presented in Table I. Our study included 58 patients, 18 men and 40 women. In the majority of patients ( $n=44,75.9 \%$ ), coronary arteries were considered normal, while the rest (7 in group A, 7 in group P) had $<50 \%$ stenotic coronary atherosclerosis. Nonsignificant atherosclerosis of the carotid arteries was present in $14 \%$ of patients $(n=8)$. More patients with arterial hypertension were in the placebo group (31 in $\mathrm{P}$ vs. 
Table I. Baseline characteristics of treatment groups

\begin{tabular}{|c|c|c|c|c|}
\hline Variable & All $(n=58)$ & $\begin{array}{l}\text { Placebo } \\
\text { group } \\
(n=31)\end{array}$ & $\begin{array}{l}\text { Atorvastatin } \\
\text { group } \\
(n=27)\end{array}$ & $P$-value \\
\hline Age [years] & $61.6 \pm 9.6$ & $59.7 \pm 9.3$ & $63.7 \pm 9.6$ & 0.062 \\
\hline Weight [kg] & $79.5 \pm 14.4$ & $79.7 \pm 15.1$ & $79.2 \pm 13.8$ & 0.963 \\
\hline Height $[\mathrm{cm}]$ & $165.1 \pm 8.6$ & $166.0 \pm 9.0$ & $164.1 \pm 8.2$ & 0.507 \\
\hline Body mass index $\left[\mathrm{kg} / \mathrm{m}^{2}\right]$ & $29.1 \pm 4.4$ & $28.8 \pm 4.2$ & $29.4 \pm 4.6$ & 0.533 \\
\hline Waist circumference $[\mathrm{cm}]$ & $96.1 \pm 11.2$ & $96.3 \pm 11.3$ & $95.8 \pm 11.2$ & 0.892 \\
\hline Systolic blood pressure [mm Hg] & $134.4 \pm 17.1$ & $134.2 \pm 18.0$ & $134.6 \pm 16.3$ & 0.870 \\
\hline Diastolic blood pressure [mm Hg] & $77.5 \pm 10.0$ & $77.0 \pm 9.6$ & $78.1 \pm 10.6$ & 0.845 \\
\hline Left ventricular ejection fraction (\%) & $59.7 \pm 2.0$ & $60.0 \pm 0.0$ & $59.4 \pm 2.9$ & 0.284 \\
\hline Male & $18(31.0 \%)$ & $11(35.5 \%)$ & $7(25.9 \%)$ & 0.433 \\
\hline Family history of cardiovascular disease & $24(41.4 \%)$ & $11(35.5 \%)$ & $13(48.2 \%)$ & 0.329 \\
\hline Smoking history & $9(15.5 \%)$ & $6(19.4 \%)$ & $3(11.1 \%)$ & 0.387 \\
\hline Arterial hypertension & $52(89.7 \%)$ & $31(100.0 \%)$ & $21(77.8 \%)$ & 0.006 \\
\hline Gastrointestinal symptomatology in the past & $20(34.5 \%)$ & $10(32.3 \%)$ & $10(37.0 \%)$ & 0.702 \\
\hline Stressful life (subjective patient assessment) & $22(37.9 \%)$ & $8(25.8 \%)$ & $14(51.9 \%)$ & 0.041 \\
\hline Anxiety or depression & $11(19.0 \%)$ & $5(16.1 \%)$ & $6(22.2 \%)$ & 0.555 \\
\hline Myocardial ischemia confirmed by ergometry & $44(75.9 \%)$ & $24(77.4 \%)$ & $20(74.1 \%)$ & 0.675 \\
\hline Myocardial ischemia confirmed by stress scintigraphy & $14(24.1 \%)$ & $7(22.6 \%)$ & $7(25.9 \%)$ & 0.766 \\
\hline
\end{tabular}

Values are expressed as mean \pm standard deviation or as number (and percentage) of patients.

21 in $\mathrm{A}, p=0.0006$ ), while hypertension was well controlled among the whole study population. The two study groups were also not homogeneous in terms of stress; about a third of the patients (more in group A) assessed their life as stressful.

The long list of study exclusion criteria was aimed at avoiding the potential confounding influence of the most important metabolic disorders such as diabetes, hypothyroidism, chronic liver and/or kidney disease. Due to its high prevalence, obesity did not qualify as an exclusion criterion, to a great extent because of the limited pool of study patients. This is one of the reasons that the majority of patients had increased waist circumference and body mass index (BMI) $\left(>30 \mathrm{~kg} / \mathrm{m}^{2}\right.$ in $23 \mathrm{pa}-$ tients (11 in group P, 12 in group A; the differences were not statistically significant).

At the beginning of the study all patients were advised to maintain a healthy lifestyle, including regular physical activity, a Mediterranean diet, and weight loss for those with a high BMI. Unfortunately, according to patients' reporting during the study, regular physical activity (defined as moderate aerobic exercise for at least $2.5 \mathrm{~h} /$ week) was only undertaken by $29.3 \%$ of patients, with no differences between the study groups. During the study none of the participants received dietary supplements, vitamin preparations or other known substances which could affect the endothelial function. Drinking alcohol was not desirable, and it was forbidden to drink more than $20 \mathrm{~g} /$ day for men and $10 \mathrm{~g}$ /day of alcohol for women. Drinking coffee or caffeinated products was also not allowed, and for at least $24 \mathrm{~h}$ before taking measurements during the study visits study participants were only allowed to drink water.

A positive family history of cardiovascular disease was present in $41 \%$ of patients, and approximately one third of patients had digestive system problems in the past; there were no statistically significant differences between groups.

At the study entry (SVO) the total, low-density lipoprotein (LDL) and non-high-density lipoprotein (HDL) cholesterol levels were increased, while triglycerides were at the recommended range upper limit according to current guidelines. At each of the subsequent study visits we found significantly lower blood lipid levels in group A (Table II, Figure 1). Other hematological and biochemical laboratory tests did not show statistically significant differences between the study groups.

The majority of patients had brachial artery endothelial dysfunction at the study entry. Significant improvement of endothelial function assessed by FMD at SV1 and SV2 was found in the atorvas- 
Table II. Blood lipid parameters and the differences between the study groups during the entire study period

\begin{tabular}{|lcccccc|}
\hline Variable & \multicolumn{2}{c}{ Atorvastatin group $(n=27)$} & \multicolumn{3}{c|}{ Placebo group $(n=31)$} \\
\cline { 2 - 7 } & SV0 & SV1 & SV2 & SV0 & SV1 & SV2 \\
\hline Total cholesterol & $5.91 \pm 0.95$ & $4.06 \pm 0.75^{\star}$ & $4.17 \pm 0.82^{\star}$ & $5.93 \pm 1.10$ & $5.58 \pm 0.96$ & $5.77 \pm 0.09$ \\
\hline LDL cholesterol & $3.77 \pm 0.73$ & $2.09 \pm 0.67^{\star}$ & $2.10 \pm 0.81^{*}$ & $3.74 \pm 1.08$ & $3.65 \pm 1.00$ & $3.61 \pm 0.82$ \\
\hline Non-HDL cholesterol & $4.56 \pm 0.96$ & $2.68 \pm 0.76^{\star}$ & $2.77 \pm 0.84^{\star}$ & $4.47 \pm 1.25$ & $4.33 \pm 1.22$ & $4.27 \pm 1.01$ \\
\hline HDL cholesterol & $1.36 \pm 0.22$ & $1.38 \pm 0.26$ & $1.41 \pm 0.27$ & $1.46 \pm 0.39$ & $1.45 \pm 0.37$ & $1.50 \pm 0.39$ \\
\hline Triglycerides & $1.73 \pm 0.95$ & $1.28 \pm 0.53$ & $1.46 \pm 0.92$ & $1.60 \pm 0.70$ & $1.48 \pm 0.76$ & $1.47 \pm 0.63$ \\
\hline
\end{tabular}

All values are expressed as mean \pm standard deviation (SD). SVO - initial visit, SV1 - $1^{\text {st }}$ control study visit at 3 months, SV2 - $2^{\text {nd }}$ study visit at 6 months of follow-up. ${ }^{\star} P$-value $<0.001$ (in comparison to placebo).

A

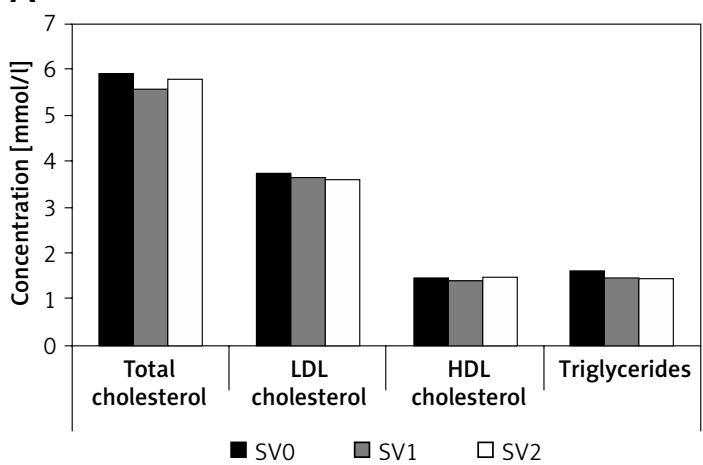

B

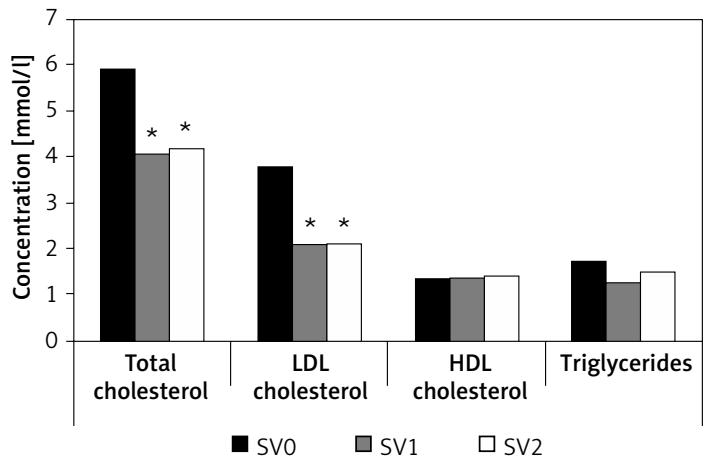

Figure 1. Lipid profile changes during the study: A - placebo group, B - atorvastatin group

SVO - initial visit, SV1 - $1^{\text {st }}$ control study visit at 3 months, SV $2-2^{\text {nd }}$ study visit at 6 months of follow-up; ${ }^{*} p$-value $<0.001$ (in comparison to placebo).

tatin treated group $(p<0.05)$, but the values of FMD achieved still did not meet the criteria of normal endothelial function (Table III, Figure $2 \mathrm{~A}$ ). No change of endothelial function was detected in group $\mathrm{P}$ during the follow-up. Also, no significant difference in endothelium-independent vasodilatation (NMD) was found between the two groups, either at inclusion or during subsequent visits (Table III, Figure 2 B).

The $\mathrm{RHI}$ was within the normal range in $59 \%$ of patients at the beginning of the study. During the follow-up no significant improvement of RHI was found in those outside the normal range at the beginning, as well as no differences between the two study groups (Table III, Figure 2 C). Rate-normalized Al showed an improvement $(-114.49 \%$ group A vs. $-30.77 \%$ group $P, p=0.077$ ) although there was no significant differences in the Al between the groups (Table III, Figures 2 D, E).

According to the SAQ, an improvement was found in almost all observed variables in both groups, with no significant differences between

Table III. Percent change in flow-mediated dilation (FMD), nitroglycerine-mediated dilation (NMD), reactive hyperemia index (RHI), augmentation index (Al) and Al/75 (augmentation index normalized to heart rate 75/min) related to baseline values during the study: SV1 $-1^{\text {st }}$ control study visit at 3 months, SV2 $-2^{\text {nd }}$ study visit at 6 months of follow-up

\begin{tabular}{|lcccc|}
\hline Parameter & \multicolumn{2}{c|}{ Atorvastatin group $(n=27)$} & \multicolumn{2}{c|}{ Placebo group $(n=31)$} \\
\cline { 2 - 5 } & SV1 & SV2 & SV1 & SV2 \\
\hline FMD & $+120.8^{*}$ & $+70.8^{*}$ & -21.2 & -1.9 \\
\hline NMD & -7.1 & -5.7 & -2.7 & +2.7 \\
\hline RHI & 0.0 & +5.0 & +5.3 & -5.3 \\
\hline Al & +7.4 & -26.5 & +27.7 & +25.5 \\
\hline Al/75 & -36.2 & $-114.5^{* *}$ & -46.2 & -30.8 \\
\hline
\end{tabular}

${ }^{*} p$-value $<0.05$ (in comparison to placebo), ${ }^{* *} p$-value $=0.077$ (in comparison to placebo). 
A

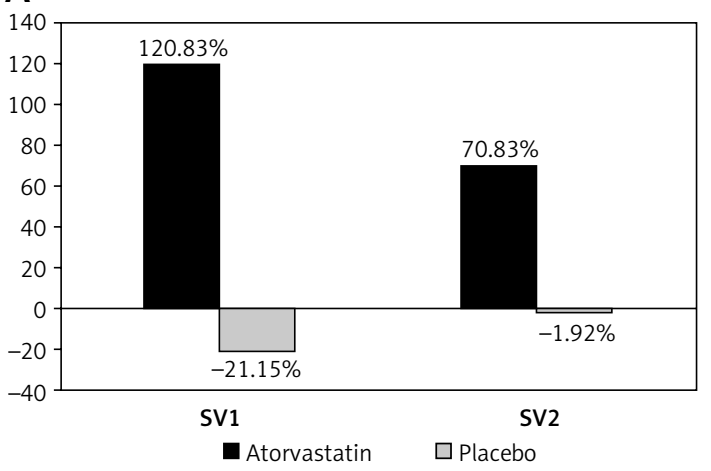

C

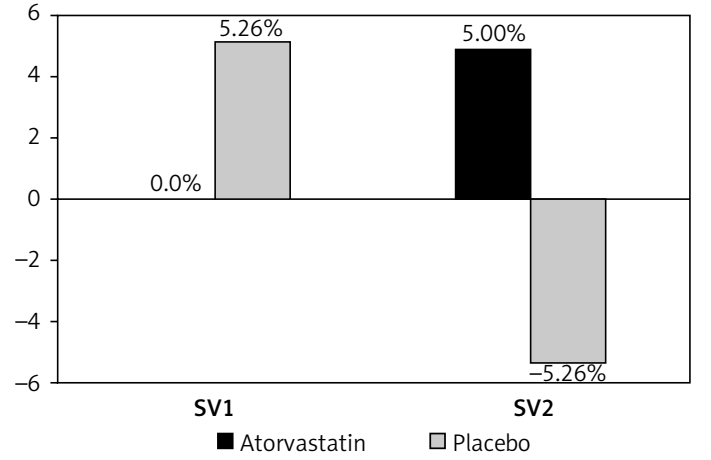

E

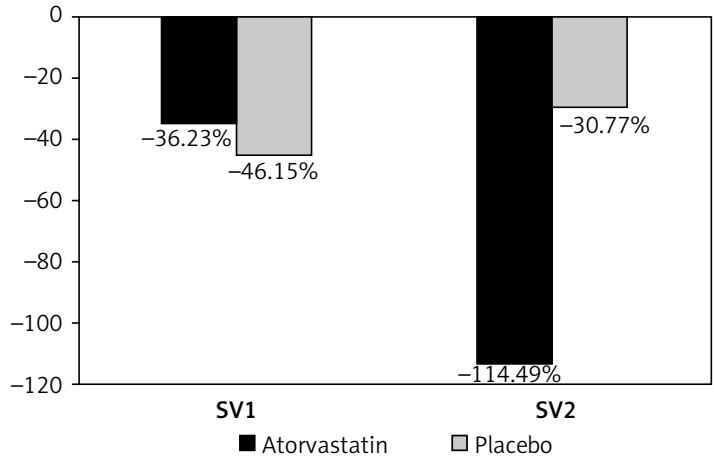

the two (Table IV). The exception is the issue of quality of life (QoL), where patients in both groups assessed their QoL at the control study visits as poorer compared with baseline. The exception was the issue of quality of life (QoL), where patients in both groups assessed their QoL at the control study visits as poorer compared with baseline. The deterioration in QoL was particularly large in group $\mathrm{P}$ and was significant at SV2 in group $\mathrm{P}$.

\section{Discussion}

The pathophysiology of AP in patients with noninvasively proven myocardial ischemia and normal coronary angiogram is not completely understood. Functional impairment of the coronary microvasculature causing an abnormal coronary flow reserve is the generally suspected mechanism [20, $21]$. In the present double-blind, randomized and
B

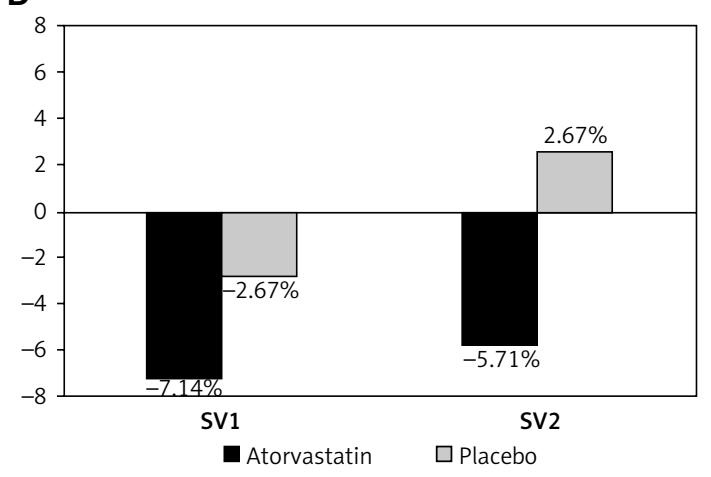

D

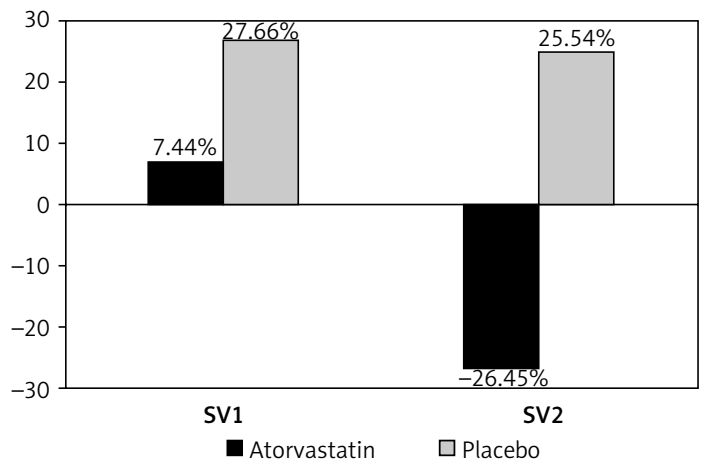

Figure 2. Endothelial function (expressed as \% change from baseline values) during the study follow-up. A - flow-mediated dilation (FMD), B - nitroglycerine-mediated dilation (NMD), $\mathrm{C}$ - reactive hyperemia index (RHI), D - augmentation index (AI), and $\mathbf{E}-\mathrm{Al} / 75$ (augmentation index normalized to heart rate $75 / \mathrm{min}$ )

SV $1-1^{\text {st }}$ control study visit at 3 months, SV $2-2^{\text {nd }}$ study visit at 6 months of follow-up.

placebo-controlled interventional pilot study we found that in this group of patients treatment with a moderate dose of atorvastatin ( $20 \mathrm{mg} /$ day) significantly improves endothelial function at the level of large conduit arteries, while at the same time no correlation was found with the potential effects on peripheral microcirculatory endothelial functioning.

The present study reports for the first time the positive vascular effects of a moderate dose of atorvastatin in patients with AP and a normal coronary angiogram. Previous studies demonstrated that statins, in parallel with their lipid-lowering effects, exert additional, pleiotropic effects, including significant endothelial function improvement, inhibition of inflammation, and stabilization of atherosclerotic plaques [10-14, 22]. In our study, atorvastatin significantly improved FMD at the 
Table IV. Seattle Angina Questionnaire (SAQ) scores and the differences between the study groups during the entire study period

\begin{tabular}{|lcccccc|}
\hline \multirow{2}{*}{ Variable } & \multicolumn{2}{c}{ Atorvastatin group $(n=27)$} & \multicolumn{2}{c|}{ Placebo group $(n=31)$} \\
\cline { 2 - 7 } & SV0 & SV1 & SV2 & SV0 & SV1 & SV2 \\
\hline Physical limitation & $88.53 \pm 10.10$ & $92.68 \pm 7.90$ & $93.75 \pm 7.85$ & $79.77 \pm 13.26$ & $86.47 \pm 12.52$ & $89.73 \pm 8.56$ \\
\hline Angina stability & $61.81 \pm 25.29$ & $72.92 \pm 23.47$ & $72.91 \pm 23.47$ & $66.13 \pm 26.70$ & $67.74 \pm 22.33$ & $66.67 \pm 23.96$ \\
\hline Angina frequency & $83.33 \pm 13.68$ & $83.68 \pm 14.84$ & $86.46 \pm 12.24$ & $83.60 \pm 14.83$ & $86.56 \pm 12.67$ & $87.10 \pm 14.57$ \\
\hline Treatment satisfaction & $47.25 \pm 17.74$ & $50.72 \pm 12.51$ & $51.01 \pm 7.14$ & $53.98 \pm 20.70$ & $57.42 \pm 12.49$ & $53.98 \pm 11.46$ \\
\hline Quality of life & $51.25 \pm 18.25$ & $49.58 \pm 19.89$ & $47.50 \pm 17.51$ & $54.19 \pm 17.47$ & $47.74 \pm 18.92$ & $46.45 \pm 15.39^{*}$ \\
\hline
\end{tabular}

All values are expressed as mean \pm standard deviation (SD). SVO - initial visit, SV1 - $1^{\text {st }}$ control study visit at 3 months, SV2 - $2^{\text {nd }}$ study visit at 6 months of follow-up. ${ }^{*}$-value $<0.05$.

level of large conduit arteries (in the range of $+70 \%$ to $+120 \%$ ). Smaller studies previously showed comparable improvement of brachial FMD in similar groups of patients treated with either pravastatin or simvastatin. In a single-blinded placebo-controlled study, Kayikcioglu et al. demonstrated a beneficial effect of pravastatin (40 mg/ day for 3 months) on FMD in 20 normolipemic patients with $\mathrm{AP}$, a positive exercise test and a normal coronary angiogram in the absence of coronary artery spasm, determined by hyperventilation. The authors presumed that this beneficial effect was probably the result of improved endothelial function [23]. Fabian et al. found significant improvement of FMD and longer time to $>1-\mathrm{mm}$ ECG ST-segment depression during stress testing $(p<0.0001)$ in hypercholesterolemic patients with AP and a normal coronary angiogram treated with simvastatin (20 patients randomized to placebo and 20 to simvastatin $20 \mathrm{mg} /$ day group) [24]. Baller et al. reported improvement of coronary flow reserve in patients with normal $(n=9)$ or slightly abnormal $(n=9)$ coronary angiograms (minimal disease with luminal irregularities and/or diameter reduction $<30 \%$ ), and moderately elevated LDL-C levels after 6-month lipid-lowering therapy with simvastatin [25]. Fluvastatin improved coronary flow reserve, endothelial function and exercise tolerance in patients with cardiac syndrome $X$, and its beneficial effects were related to the elevation of nitric oxide and reduction of endothelin-1 [26].

In our study the significant endothelial function improvement induced by atorvastatin was already expressed after the first 3 months of treatment, while prolonged treatment for 6 months did not result in further FMD increase or its full normalization. The most likely reason is the use of only a moderate dose of the drug, since available data suggest that more intensive therapy with higher doses of atorvastatin (40 or $80 \mathrm{mg} /$ day) is associated with more potent pleiotropic effects [27-29]. However, other authors before also reported posi- tive effects of low doses of atorvastatin on the microcirculation. Holowatz et al. observed the beneficial effects of low-dose atorvastatin $(10 \mathrm{mg} /$ day for 3 months) on NO-dependent vasodilatation of cutaneous microvasculature in hypercholesterolemic patients [30, 31].

Endothelial dysfunction measured by FMD was found to be a marker of early atherosclerotic changes of the arterial circulation, including coronary arteries [32]. On the other hand, inappropriate endothelial regulation can also be a functional disorder of coronary microcirculation. It has been demonstrated that coronary microvascular dysfunction is probably part of a more generalized vascular disorder, since it is often associated with endothelial dysfunction of the peripheral conduit arteries and smooth muscle cell dysfunction in other organs [33-37]. To the best of our knowledge, to date, the changes in endothelial function at the level of the peripheral microcirculation in patients with angina and normal coronary angiogram have not been investigated. In our study the microcirculatory endothelial function was assessed by peripheral arterial tonometry (PAT) simultaneously with FMD measurements.

While at the study entry endothelial dysfunction assessed by brachial artery FMD was found in the great majority of our patients, the markers of microcirculatory endothelial function of digital arteries (assessed by PAT), such as RHI, were found to be still in the normal range in almost $60 \%$ of the patients. It was demonstrated that vascular dysfunction registered by PAT is associated with the presence of various major risk factors and/or cardiovascular disease [38-40]. Previous studies indicated a relationship between coronary endothelial dysfunction, as detected by invasive evaluation of the coronary arteries, and the findings of PAT. It was suggested that PAT potentially offers a more simplified non-invasive measurement and less operator-dependent technique, although it measures vascular functions other than FMD [39, 41]. Moreover, it was shown that PWA predicts 
future adverse outcome events in patients with obstructive coronary artery disease [42].

In our group of patients with angina and a normal coronary angiogram a moderate dose of atorvastatin significantly improved FMD, but at the same time did not improve the endothelial function of digital arteries expressed by $\mathrm{RHI}(+5 \%$, $p=0.897)$. Although some studies have demonstrated a direct contribution of nitric oxide to both the brachial artery FMD and digital PAT values, conflicting results regarding the correlation between these two investigative methods have been reported [7]. Similarly, our findings confirm that PAT and brachial artery FMD probably assess functions of somewhat distinct vascular segments [43]. On the other hand, the inconsistency of the results obtained using different measurement techniques could reflect either the evaluation of different pathophysiological aspects of the disease, or in addition to those already known, some still unrecognized factors expressed more in only one of the evaluated arterial tree segments. For example, Stulc et al. suggested that dysfunction of the microcirculation in patients with hypercholesterolemia appears later in the course of atherosclerotic disease [44].

Further research is needed to better explain the relations between endothelial function at the level of large conduit (including coronary) arteries and coronary microcirculation, as well as the pharmacological effects on its improvement. However, currently only invasive techniques (using quantitative coronary angiography and/or intravascular ultrasound) involving local delivery of vasoactive agents are available for this purpose [45].

Using PAT we found an improvement of the Al normalized to a rate of $75 / \mathrm{min}$ in patients treated with atorvastatin compared with placebo $(p=$ 0.077). Such a finding supports the previously reported beneficial effect of atorvastatin on arterial stiffness and could reflect its positive actions on the vasculature beyond its lipid-lowering properties [46].

In our study we also evaluated the potential effect of atorvastatin treatment on the quality of life in patients with angina and a normal coronary angiogram. Using a standardized questionnaire (SAQ) we did not find any significant difference in various evaluated dimensions between the two study groups (atorvastatin or placebo). Pizzi et al. showed that 6 months of combined therapy with atorvastatin and ramipril improved not only endothelial function measured by FMD but also quality of life, determined by the SAQ score [47]. We found a trend in improvement in almost all observed variables in both study groups. Among the possible reasons for such a finding could be the simultaneous quite homogeneous comprehensive medical treatment with other vasoactive drugs in both groups (including bisoprolol, amlodipine and aspirin), better control of other major risk factors, such as arterial hypertension, more regular physical activity, and more frequent physician visits.

The most important limitation of our study is that spontaneous and/or pharmacologically induced spasms of the coronary arteries were not excluded in the patients enrolled. Intracoronary testing is not only technically challenging, it is quite time-consuming, expensive and carries additional risk for patients. One possible weakness of the study is the inclusion of patients with angiographically present nonobstructive atherosclerotic coronary artery disease, despite the fact that the two study groups did not differ in this regard. We have to be aware of the general limitation of coronary angiography in defining a normal coronary angiogram, since it provides only information about the contours of the vascular lumen and not a 3-dimensional view of the vessel wall. More information on its structure and disease changes can be obtained using more modern methods such as intravascular ultrasound, optical coherence tomography and coronary angioscopy, which were not used in our study. Furthermore, obesity and arterial hypertension per se, both well-known major CVD risk factors associated with endothelial dysfunction, were not listed among the exclusion criteria [48, 49]. Among the reasons for this decision is their high prevalence in patients with $\mathrm{AP}$, and that numerous other exclusion criteria already significantly reduced the number of potentially eligible patients for the study. Since there were no differences between the groups regarding obesity, we trust it did not importantly influence our results. On the other hand, patients with uncontrolled arterial hypertension were excluded, while at the same time we ensured quite homogeneous treatment of arterial hypertension for all enrolled patients with the study drugs used. By combining a $\beta$-blocker (bisoprolol) and calcium channel blocker (amlodipine) we also tried to avoid their potentially confounding effects in case they would be used only by a limited number of study participants.

We would also like to emphasize the strengths of our study. First and the foremost, this was a double-blinded and placebo-controlled study. Second, due to the numerous exclusion criteria, the study group was quite homogeneous, and potentially confounding factors affecting endothelial function were reduced. Finally, the effects of atorvastatin were evaluated not only in terms of lipid lowering and endothelial function improvement but also with regard to the different aspects of the quality of life of patients with AP and a normal coronary angiogram. 
In conclusion, in our double-blind, placebo-controlled, randomized pilot study, a statistically significant difference in FMD of the brachial artery in patients treated with atorvastatin compared with placebo was found. This suggests that atorvastatin has a favorable effect on the endothelial function of large conduit arteries in patients with AP and a normal coronary angiogram and probably has positive effects on the endothelial function of coronary arteries in these patients. On the other hand, no correlation between the measurement of endothelial function by PAT and brachial artery FMD was found. Our findings suggest that PAT and brachial artery FMD assess the endothelial function of distinct vascular segments and are not comparable.

\section{Acknowledgments}

The study was performed as part of the research program P3-0308, supported by the Ministry for Education, Science and Technology of the Republic of Slovenia. The authors also gratefully acknowledge the contribution of the pharmaceutical company Lek Sandoz (from Ljubljana, Slovenia), which kindly prepared and provided the study drugs.

\section{Conflict of interest}

The authors declare no conflict of interest.

\section{References}

1. Titterington JS, Hung OY, Wenger NK. Microvascular angina: an update on diagnosis and treatment. Future Cardiol 2015; 11: 229-42.

2. Marinescu MA, Löffler Al, Ouellette M, et al. Coronary microvascular dysfunction, microvascular angina, and treatment strategies. JACC Cardiovasc Imaging 2015; 8: 210-20.

3. Kaski JC, Iqbal K. Cardiac syndrome X: pathogenesis and management. Heart Metab 2008; 40: 30-5.

4. Yang EH, Lerman A. Angina pectoris with a normal coronary angiogram. Herz 2005; 30: 17-25.

5. Schachinger V, Britten MB, Zeiher AM. Prognostic impact of coronary vasodilator dysfunction on adverse long-term outcome of coronary heart disease. Circulation 2000; 101: 1899-906.

6. Gokce N, Keaney JR Jr, Hunter LM, et al. Risk stratification for postoperative cardiovascular events via noninvasive assessment of endothelial function: a prospective study. Circulation 2002; 105: 1567-72.

7. Poredos P, Jezovnik MK. Testing endothelial function and its clinical relevance. J Atheroscler Thromb 2013; 20: 1-8.

8. Versari D, Daghini E, Virdis A, Ghiadoni L, Taddei S. Endothelial dysfunction as a target for prevention of cardiovascular disease. Diabetes Care 2009; Suppl 2: S314-21.

9. Hognestad A, Aukrust P, Wergeland R, et al. Effects of conventional and aggressive statin treatment on markers of endothelial function and inflammation. Clin Cardiol 2004; 27: 199-203.
10. Davignon J. Beneficial cardiovascular pleiotropic effects of statins. Circulation 2004; 109: 39-43.

11. Sahebkar A, Kotani K, Serban C, et al. Statin therapy reduces plasma endothelin-1 concentrations: a metaanalysis of 15 randomized controlled trials. Atherosclerosis 2015; 241: 433-42.

12. Serban C, Sahebkar A, Ursoniu S, et al. A systematic review and meta-analysis of the effect of statins on plasma asymmetric dimethylarginine concentrations. Sci Rep 2015; 5: 9902.

13. Katsiki N, Mikhailidis DP, Banach M. Effects of statin treatment on endothelial function, oxidative stress and inflammation in patients with arterial hypertension and normal cholesterol levels. J Hypertens 2011; 29: 2493-4.

14. Kowalski J, Barylski M, Banach M, Grycewicz J, Irzmański R, Pawlicki L. Neutrophil superoxide anion generation during atorvastatin and fluvastatin therapy used in coronary heart disease primary prevention. J Cardiovasc Pharmacol 2006; 48: 143-7.

15. Oikonomou E, Siasos G, Zaromitidou M, et al. Atorvastatin treatment improves endothelial function through endothelial progenitor cells mobilization in ischemic heart failure patients. Atherosclerosis 2015; 238: 159-64.

16. Katsiki N, Doumas M, Mikhailidis DP. Lipids, statins and heart failure: an update. Curr Pharm Des 2016; 22: 4796-806.

17. Bielecka-Dabrowa A, Mikhailidis DP, Rizzo M, von Haehling S, Rysz J, Banach M. The influence of atorvastatin on parameters of inflammation left ventricular function, hospitalizations and mortality in patients with dilated cardiomyopathy: 5-year follow-up. Lipids Health Dis 2013; 12: 47.

18. Bielecka-Dabrowa A, Goch JH, Rysz J, et al. Influence of co-existing atrial fibrillation on the efficacy of atorvastatin treatment in patients with dilated cardiomyopathy: a pilot study. Lipids Health Dis 2010; 9: 21.

19. Celermajer DS, Sorensen KE, Bull C, Robinson J, Deanfield JE. Endothelium-dependent dilation in the systemic arteries of asymptomatic subjects relates to coronary risk factors and their interaction. J Am Coll Cardiol 1994; 24: 1468-74.

20. Maseri A, Crea F, Kaski JC, Crake T. Meshanisms of angina pectoris in syndrome X. J Am Coll Cardiol 1991; 17: 499-506.

21. Meeder JG, Blanksma PK, Crijns HJGM, et al. Mechanism of angina pectoris in syndrome- $X$ assessed by myocardial perfusion dynamics and heart rate variability. Eur Heart J 1995; 16: 1571-7.

22. Tomás JP, Moya JL, Campuzano R, et al. Noninvasive assessment of the effect of atorvastatin on coronary microvasculature and endothelial function in patients with dyslipidemia. Rev Esp Cardiol 2004; 57: 909-15.

23. Kayikcioglu M, Payzin S, Yavuzgil O, Kultzrsay H, Can LH, Soydan I. Benefits of statin treatment in cardiac syndrome-X. Eur Heart J 2003; 24: 1999-2005.

24. Fábián E, Varga A, Picano E, Vajo Z, Rónaszéki A, Csanády $M$. Effect of simvastatin on endothelial function in cardiac syndrome X patients. Am J Cardiol 2004; 94: 652-5.

25. Baller D, Gleichmann U, Notohamiprodjo G, et al. Improved coronary vasodilator capacity by drug lipid lowering therapy in patients in the early stage of coronary atherosclerosis with reduced coronary reserves and moderate LDL hypercholesteremia. Z Kardiol 1998; 87 Suppl 2: 136-44.

26. Zhang X, Li Q, Zhao J, et al. Effects of combination of statin and calcium channel blocker in patients with cardiac syndrome X. Coron Artery Dis 2014; 25: 40-4. 
27. Ray KK, Cannon CP. Atorvastatin and cardiovascular protection: a review and comparison of recent clinical trials. Expert Opin Pharmacother 2005; 6: 915-27.

28. Bielecka-Dabrowa A, Goch JH, Mikhailidis DP, Rysz J, Maciejewski $M$, Banach $M$. The influence of atorvastatin on parameters of inflammation and function of the left ventricle in patients with dilated cardiomyopathy. Med Sci Monit 2009; 15: MS12-23.

29. Lee BK, Koo BK, Nam CW, et al. Does pre-treatment with high dose atorvastatin prevent microvascular dysfunction after percutaneous coronary intervention in patients with acute coronary syndrome? Korean Circ J 2016; 46: 472-80.

30. Holowatz LA, Kenney WL. Acute localized administration of tetrahydrobiopterin and chronic systemic atorvastatin treatment restore cutaneous microvascular function in hypercholesterolaemic humans. J Physiol 2011; 589: 4787-97.

31. Holowatz LA, Santhanam L, Webb A, Berkowitz DE, Kenney WL. Oral atorvastatin therapy restores cutaneous microvascular function by decreasing arginase activity in hypercholesterolaemic humans. J Physiol 2011; 589 : 2093-103.

32. Gimbrone MA Jr, García-Cardeña G. Endothelial cell dysfunction and the pathobiology of atherosclerosis. Circ Res 2016; 118: 620-36.

33. Lekakis JP, Papamichael CM, Vemmos CN, et al. Peripheral vascular endothelial dysfunction in patients with angina pectoris and normal coronary arteriograms. J Am Coll Cardiol 1998; 31: 541-6.

34. Masci PG, Laclaustra M, Lara JG, Kaski JC. Brachial artery flow-mediated dilation and myocardial perfusion in patients with cardiac syndrome X. Am J Cardiol 2005; 95: 1478-80

35. Cannon RO $3^{\text {rd }}$, Peden DB, Berkebile C, et al. Airway hyperresponsiveness in patients with microvascular angina. Evidence for a diffuse disorder of smooth muscle responsiveness. Circulation 1990; 82: 2011-7.

36. Weidmann B, Jansen WC, Bock A, et al. Technetium 99m-HMPAO brain SPECT in patients with syndrome $X$ Am J Cardiol 1997; 79: 959-61.

37. Atmaca Y, Ozdemir AO, Ozdol C, et al. Angiographic evaluation of myocardial perfusion in patients with syndrome X. Am J Cardiol 2005; 96: 803-5.

38. Hamburg NM, Keyes MJ, Larson MG, et al. Cross-sectional relations of digital vascular function to cardiovascular risk factors in the Framingham Heart Study. Circulation 2008; 117: 2467-74

39. Bonetti PO. Attenuation of digital reactive hyperemia in patients with early and advanced coronary artery disease. JACC 2005; 45 Suppl.: 407.

40. Kuvin JT, Patel AR, Sliney KA, et al. Assessment of peripheral vascular endothelial function with finger arterial pulse wave amplitude. Am Heart J 2003; 146: 168-74.

41. Woo JS, Jang WS, Kim HS, et al. Comparison of peripheral arterial tonometry and flow-mediated vasodilation for assessment of the severity and complexity of coronary artery disease. Coron Artery Dis 2014; 25: 421-6.

42. Kitta Y, Obata JE, Nakamura T, et al. Persistent impairment of endothelial vasomotor function has a negative impact on outcome in patients with coronary artery disease. J Am Coll Cardiol 2009; 53: 323-30.

43. Lee CR, Bass A, Ellis K, et al. Relation between digital peripheral arterial tonometry and brachial artery ultrasound measures of vascular function in patients with coronary artery disease and in healthy volunteers. Am J Cardiol 2012; 109: 651-7.
44. Stulc T, Kasalová Z, Prázný M, Vrablík M, Skrha J, Ceska R. Microvascular reactivity in patients with hypercholesterolemia: effect of lipid lowering treatment. Physiol Res 2003; 52: 439-45.

45. Cox DA, Vita JA, Treasure CB, et al. Atherosclerosis im pairs flow-mediated dilation of coronary arteries in hu mans. Circulation 1989; 80: 458-65.

46. Kanaki Al, Sarafidis PA, Georgianos PI, et al. Effects of low-dose atorvastatin on arterial stiffness and central aortic pressure augmentation in patients with hypertension and hypercholesterolemia. Am J Hypertens 2013; 26: 608-16.

47. Pizzi C, Manfrini O, Fontana F, Bugiardini R. Angiotensinconverting enzyme inhibitors and 3-hydroxy-3-methylglutaryl coenzyme A reductase in cardiac syndrome $X$. Role of superoxide dismutase activity. Circulation 2004; 109: 53-8.

48. Goodwill AG, Frisbee SJ, Stapleton PA, James ME, Frisbee JC. Impact of chronic anticholesterol therapy on development of microvascular rarefaction in the metabolic syndrome. Microcirculation 2009; 16: 667-84.

49. Clough GF, Turzyniecka M, Walter L, et al. Muscle microvascular dysfunction in central obesity is related to muscle insulin insensitivity but is not reversed by highdose statin treatment. Diabetes 2009; 58: 1185-91. 